\title{
MÉTODOS DE SINCRONIZACIÓN DE CELO EN BOVINOS DE LECHE APLICABLES PARA LA MESETA DE POPAYÁN
}

\author{
METHODS OF SYNCHRONIZATION OF HEAVEN IN MILK BOVES \\ APPLICABLE FOR THE POPAYAN TABLE
}

\begin{abstract}
Yesid Eduardo Bastidas Vallejo
Zootecnia, Universidad Nacional Abierta y a Distancia djyesidbastidas@hotmail.com
\end{abstract}

\section{Manuel Emilio Gómez Candel}

Zootecnista. Candidato a Magister Maestría en Desarrollo Alternativo Sostenible y Solidario, Universidad Nacional Abierta y a Distancia manuel.gomez@unad.edu.co

\section{RESUMEN.}

El presente artículo tiene como objetivo, conceptualizar sobre los distintos métodos de sincronización de celo en bovinos de leche, para ser usados como referencia por estudiantes, profesionales y empresarios ganaderos, presentado como alternativa de mejoramiento genético en sus hatos la implementación de un programa de sincronización bovina.

Reseñándose métodos y protocolos conocidos tanto a nivel internacional, como nacional y departamental y las ventajas y desventajas de su aplicación en las ganaderías en la meseta de Popayán, la forma cómo se aplica, las razas que mejor asimilan este tratamiento y las más apropiadas. Se busca entonces enriquecer la experiencia académica e investigativa, y que sirva de referente para la aplicación de estas técnicas en las diferentes ganaderías de Popayán.

Palabras Clave: Sincronización, protocolos, técnica, reproducción, mejoramiento genético, hatos lecheros.

DOI: https://doi.org/10.22490/26653176.3559 


\section{ABSTRACT.}

The purpose of this article is to conceptualize the different methods of heat synchronization in milk cattle, to be used as a reference by students, professionals and livestock entrepreneurs, presented as an alternative for genetic improvement in their herds the implementation of a synchronization program bovine

Outlining known methods and protocols both internationally, nationally and departmentally, and the advantages and disadvantages of their application in livestock farms on the Popayán plateau, the manner in which they are applied, the breeds that best assimilate this treatment and those most appropriate for Popayan plateau. It is sought then to enrich the academic and research experience, and to serve as a reference for the application of these techniques in the different livestock of Popayán

Keywords: Synchronization, protocols, technique, reproduction, genetic improvement, dairy herds.

\section{INTRODUCCIÓN.}

Con la presente revisión se pretende conceptualizar sobre los métodos más comunes de sincronización de celo en bovinos de leche, siendo un documento de referencia donde se analiza la implementación de la sincronización bovina como alternativa de mejoramiento genético en los hatos ganaderos.

Según Nebel y Jobst (1998), el principal factor que incide en el rendimiento reproductivo del ganado bovino, son las fallas presentadas en la detección de celo en una forma eficiente y precisa, que permita realizar una oportuna inseminación y así lograr una buena eficiencia reproductiva en el hato ganadero.

Se realiza un repaso bibliográfico sobre el tema, reseñándose métodos y protocolos de sincronización de celo conocidos tanto a nivel internacional, 
nacional y regional con las ventajas y desventajas para su aplicación en las ganaderías en la meseta de Popayán Cauca, la forma cómo se aplican, para concluir cuales son los métodos más apropiados para la meseta de Popayán.

\section{MARCO TEÓRICO}

Se trata de una investigación donde se recopilo información de publicaciones que contenían información relevante relacionadas con "métodos de sincronización de celo en bovinos de leche", a nivel general.

De las 50 reseñas bibliográficas analizadas sobre "métodos de sincronización de celo en bovinos de leche", se encontró que los métodos más conocidos y empleados en la sincronización del celo son básicamente tratamientos hormonales, que han ido evolucionando simultáneamente con los avances en el conocimiento de procesos fisiológicos que acontecen en el ovario durante el ciclo sexual de las hembras.

En la bibliografía revisada, se observa que la "sincronización de celo", es una técnica complementaria a la inseminación que "modifica los ciclos de un grupo de hembras, permitiendo que presenten un ciclo fértil en uno o unos días programados, pudiendo realizar IA, si se quiere sin detección de celos a tiempo fijo", (López, 2013). Convirtiendo esta técnica en una parte integral del manejo reproductivo en muchos hatos lecheros, dándoles a los ganaderos pautas para el mejoramiento genético de su ganado y enseñándoles al mismo tiempo a que comprendan que una pobre o inadecuada detección de celos, es la principal causa de un bajo porcentaje de inseminación y de una pobre eficiencia reproductiva en los hatos lecheros. (López, 2013).

El trabajo investigativo de Becaluba (2006), "Métodos de sincronización de celo en bovinos", hace un recuento de las diferentes propuestas y métodos conocidos ente los que se encuentran los propuestos por Christian y Casida en 1948, que surgieron con la utilización de la progesterona con el fin de bloquear la función reproductiva. "A partir de la suspensión de la medicación buena parte de los animales presentaron 
síntomas de celo. Más tarde en 1968 Wiltbank y Kasson verificaron que la adición de un estrógeno (Valerato de estradiol) al inicio del tratamiento a través de su efecto luteolítico, aumentaba la incidencia de celos en los animales tratados y permitía la reducción del periodo de bloqueo con progesterona"

Es evidente que la búsqueda para la solución de este problema de manejo llevó a idear mejores protocolos para la sincronización del celo y la ovulación, que al ser más eficientes durante el servicio permiten mantener índices de fertilidad adecuados. Idealmente, un protocolo de sincronización del estro debe producir un estro fértil y una alta respuesta desincronización, cuando es utilizado en un grupo de hembras. Colazo, (2014) concluye que: "Los protocolos de sincronización de celo y, en los últimos años, de la ovulación han adquirido gran importancia", porque los productores buscan aumentar su productividad, y una vía para ello es haciendo el proceso de reproducción más eficiente, lográndose a través del uso de protocolos de sincronización del celo y/o la ovulación que ofrezcan las mejores ventajas y mayores beneficios al productor.

Actualmente ya no se habla solamente de sincronización del celo, ahora también se habla de sincronización de la ovulación, lo cual significa que el celo y la ovulación se sincronizan con el objeto de aumentar las posibilidades de éxito del servicio. Son muchos los protocolos disponibles, sin embargo, solo algunos tratan de imitar la fisiología del animal y posiblemente son estos los que den mejores resultados (Colazo, 2007).

Para Castaño et al. (2010), por ejemplo, el uso de progestágenos puede suministrarse a través del alimento como acetato de melengestrol, implantes subcutáneos o dispositivos intravaginales. Los progestágenos bloquean el hipotálamo para simular una fase lútea, con los cuales suprime la conducta estral y la ovulación hasta que sean retirados. Estos también pueden ser usados en novillas prepúberes o vacas anestradas para inducir los celos, aunque los resultados de fertilidad han sido muy variables.

La aplicación de prostaglandinas, hacen que estas hormonas provoquen la ruptura de una estructura presente en el ovario, frenando la secreción 
de progesterona, lo que marca el fin de un ciclo estral. Y la administración de estrógeno combinado con progesterona, promueve el estro de la hembra (Cavestany,2013). Además, se pudo conocer los protocolos que sincronizan el crecimiento folicular, la ovulación, y optimizan la regresión del cuerpo lúteo dando paso de la fase luteal a la fase folicular del ciclo estral.

Es recomendable el tratamiento del DIB $®$ por manejo" en el estudio "uso de tratamientos hormonales para sincronizar el celo y la ovulación en vaquillonas (2007), estudiado por Colazo M.G. et. al, (2007), se revisa brevemente el ciclo estral, sistemas de sincronización de celos y algunos de los programas de IATF utilizados a nivel internacional, como por ejemplo Canadá, concluyendo que la mayor comprensión de la función ovárica en bovinos ha facilitado el desarrollo de protocolos para sincronizar el celo y la ovulación y que la detección de celo puede ser eliminada (o al menos minimizada) sin comprometer las tasas de preñez.

Otros estudios acerca de la sincronización de celo, llevado a cabo por varios autores, dan a conocer la importancia de estos programas o protocolos, para facilitar los procesos de inseminación artificial y disminuir los costos del control de celo, como lo enuncia Castaño Jorge et. at. (s.f.), en un estudio titulado "Comparación de dos protocolos de sincronización del celo en ganado lechero Jersey de la finca La Teresita en el municipio de Dosquebradas, Risaralda" con el objetivo de conocer la eficiencia de estos dos protocolos (sincronización de celos y ovulación en ganado lechero), concluyendo que, por la falta de estudios sobre este tema, solo se pudo "encontrar en la literatura reportada la comparación de algunos protocolos con dispositivos intravaginales como el CIDR $®$ con el Crestar $®$ donde demuestra que los resultados son iguales a los del presente estudio sin diferencias significativas entre ambos protocolos". Hallándose que los tratamientos DIB y Gestavec presentaron similar respuesta en cuanto el porcentaje de preñez al primer servicio y que la decisión de escoger un protocolo dependerá del factor económico "siendo el Gestavec la mejor opción". Estos autores, también manifiestan que el "factor manejo" es necesario tenerlo en cuenta, y que la raza estudiada (Jersey), se caracteriza por altas tasas de fertilidad, por lo que sería recomendable 


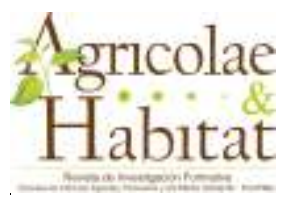

aplicarlo en otras razas, teniendo en cuenta que "para pequeños ganaderos es recomendable el tratamiento de Gestavec $₫$ por economía y para ganaderos que manejan grandes números de animales es recomendable el tratamiento del DIB $®$ por manejo". En el estudio "uso de tratamientos hormonales para sincronizar el celo y la ovulación en vaquillonas (2007), estudiado por Colazo M.G. et. al, (2007), se revisa brevemente el ciclo estral, sistemas de sincronización de celos y algunos de los programas de IATF utilizados a nivel internacional, como por ejemplo Canadá, concluyendo que la mayor comprensión de la función ovárica en bovinos ha facilitado el desarrollo de protocolos para sincronizar el celo y la ovulación y que la detección de celo puede ser eliminada (o al menos minimizada) sin comprometer las tasas de preñez.

\section{DISCUSIÓN}

Esta claramente demostrado que la eficiencia reproductiva es uno de los aspectos cruciales en la rentabilidad de las explotaciones lecheras. Sin embargo, según varios estudios e instituciones ganaderas colombianas como FEDEGAN, ICA., Contexto Ganadero. (2017), y en otros países IRAC (argentina), PESA (Nicaragua), FAO y FIL. (2012) (internacional), han coincidido en que existe un progresivo decaimiento de la fertilidad de las vacas en lactancia y el tiempo y esfuerzo que se requieren para realizar la detección de celos, han afectado los parámetros reproductivos de los rodeos lecheros en todas partes.

La mayoría de los autores, confirman que la Sincronización de celo en bovinos (de leche y doble propósito), es una herramienta que ayuda a que la explotación lechera sea más eficiente. Para muchos de ellos, la aplicación de métodos de sincronización de celos en bovinos (con la manipulación del ciclo estral), permiten la utilización de forma eficiente de la Inseminación Artificial.

La implementación de herramientas como la inseminación artificial, se presenta como una herramienta fundamental para que los ganaderos de la zona de la meseta de Popayán puedan alcanzar el mejoramiento genético de sus hatos lo que les garantizaría una mejor capacidad 
reproductiva, obtenida a través de animales con buena condición corporal y eficientes a la hora de reproducirse.

En esta zona la reproducción de los hatos está basada en su gran mayoría en un sistema de explotación tradicional, implementado estrategias que resultan insuficientes para mantener en pie la actividad, además de la falta de conocimiento en técnicas actuales y a la limitada prestación de asistencia técnica profesional, afectando la parte reproductiva generando un aumento en los días abiertos entre partos lo que trae pérdidas económicas a los ganaderos, de aquí surge la necesidad de implementar un sistema de inseminación artificial a tiempo fijo, con el fin de alcanzar avances técnicos para la zona, dicho sistema se basa en un protocolo específico, en donde se programa una determinada cantidad de hembras a ser servidas en una misma fecha, de esta manera se consigue una disminución de los días abiertos ahorrando tiempo y dinero, y nos garantizarían un mejor rendimiento en los hatos ganaderos.

Una gran ventaja que hay entre los ganaderos de la meseta de Popayán, es que cuentan con instituciones universitarias, que preparan de manera excelente e idónea a profesionales que pueden asesorar y hacer seguimiento a sus hatos ganaderos, que conocen ampliamente sobre salud y reproducción animal, y que muy seguramente contribuirán a que los programas, métodos o protocolos a aplicar, se hagan de la forma correcta. Son ellos, los profesionales los que contribuirán a que el éxito de cualquier programa reproductivo sea positivo, y puedan enseñarles a estos ganaderos, tanto las ventajas como las desventajas que tiene esta técnica.

\section{CONCLUSIONES}

Partiendo de la revisión bibliográfica realizada, se aprecia que los métodos empleados para sincronizar el celo en bovinos más importantes, son los que se basan en el uso de inyecciones periódicas de prostaglandina F2a, los que utilizan GnRH o dispositivos con progesterona, además de la adición de gonadotrofina coriónica equina (eCG) a los tratamientos con dispositivos con progesterona y estradiol, ya que todos estos métodos 


\section{Agricolae
Habitat}

posibilitan que se presenten altas tasas de preñez en vacas productoras de leche cíclicas y no cíclicas a través de la IATF. (Inseminación Artificial a Tiempo Fijo).

\section{BIBLIOGRAFÍA.}

Becaluba, F. (2006). Métodos de sincronización de celos en bovinos. Especialista en Reproducción, Buenos Aires. Argentina. Disponible en: www.produccionanimal.com.ar.

Bó, G. A. (2011). Programas de IATF en ganado bovino lechero. Instituto de Reproducción Animal Córdoba (IRAC), Paraje Pozo del Tigre, Gral. Paz (5145), Córdoba, Argentina Instituto de Ciencias Básicas, Universidad Nacional de Villa María, Córdoba, Argentina SPERMOVA. Disponible en: http://spermova.pe/site2/files/Revistas/Rev.No.1Vol1/34-43PROGRAMAS-DEIATF-EN-GANADO-BOVINO-LECHERObo2011.pdf.

Colazo, M.G., Mapletoft R.J., Martinez M.F. y Kastelic J.P. (2007). El uso de tratamientos hormonales para sincronizar el celo y la ovulación en vaquillonas. Ciencia Veterinaria. Vol 9. Núm. 1. 2007. General Pico La Pampa. República de Argentina. ISSN. 1515-1883. Disponible en: http://www. biblioteca.unlpam.edu.ar/pubpdf/revet/n09a02colazo. pdf

Federación de Ganaderos de Colombia. (2016). Recuperado de http://www.fedegan.org.co/estadisticas/publicaciones-estadisticas

Giraldo-Giraldo, J.J. (2008). Revisión de tema: Sincronización y resincronización de celos y de ovulaciones en ganado de leche y carne. Línea de Investigación: Biotecnología Pecuaria, Semillero de Investigación BIPE/ Grupo de investigación en producción, desarrollo y transformación agropecuaria. Universidad Nacional de Colombia Sede Medellín, Facultad de Ciencias Administrativas y Agropecuarias, Corporación Universitaria Lasallista Revista Lasallista de Investigación vol. 5 No 2. Print versión ISSN1794-4449

IRAC. Instituto de Reproducción Animal. Actualidades de las técnicas de super-ovulación y transferencia de embriones (2017). Córdoba 
Argentina.
http://www. iracbiogen.com.ar/front/irac. asp? NoticiaID $=19$

en:

López-López, O. (2013). Sincronización de celo en vacas. Ginecología y Obstetricia. Universidad Nacional Agraria de Nicaragua. Sede Regional Camoapa. Disponible en: http://es.slideshare.net/otonielalopez/sincronizacin-de-celos-envacas

Nebel, R. L., y Jobst, S. M. (1998). Evaluation of systematic breeding programs for lactating dairy cows: a review. Journal of Dairy Science, 81(4), 1169-1174.

Quintero Ch., E., Grajales, J.K. (2013). Tasa de concepción en vacas en lactancia tratadas con dispositivos intravaginales con diferentes concentraciones de progesterona. Universidad Tecnológica Oteima. Chiriquí, República de Panamá. Disponible en: http://www. oteima.ac.pa/nueva/wpcontent/uploads/2013/08/INV ESTIGACION-VACAS.pdf

Rivadeneira, V. (s.f.). Ciclo estral bovino. Sistema de Revisiones en Investigación Veterinaria de San Marcos. Universidad Nacional Mayor de San Marcos. Facultad de Medicina Veterinaria. Perú. Disponible en: http://veterinaria.unmsm.edu.pe/files/Articulo_ciclo_estral_bovino _rivadeneira.pdf

Scándolo, D.G., Vottero, D., Cuatrín, A., Maciel, M. (2011). Relación entre la presencia de celo y la preñez en un protocolo de sincronización de la ovulación. Instituto Nacional de Tecnología Agropecuaria (INTA), Estación Experimental Agropecuaria Rafaela, 2300 Rafaela, Argentina Pág. $325 . \quad$ Disponible en: http://www.iracbiogen.com.ar/admin/biblioteca/documentos/resu men_simposio_2011.pdf 